\title{
Redefined clinical features and diagnostic criteria in autoimmune polyendocrinopathy- candidiasis-ectodermal dystrophy
}

Elise M.N. Ferre, ${ }^{1}$ Stacey R. Rose, ${ }^{1}$ Sergio D. Rosenzweig, ${ }^{2}$ Peter D. Burbelo, ${ }^{3}$ Kimberly R. Romito, ${ }^{2}$ Julie E. Niemela, ${ }^{2}$ Lindsey B. Rosen, ${ }^{4}$ Timothy J. Break, ${ }^{1}$ Wenjuan Gu, ${ }^{5}$ Sally Hunsberger, ${ }^{6}$ Sarah K. Browne, ${ }^{4}$ Amy P. Hsu, ${ }^{4}$ Shakuntala Rampertaap, ${ }^{2}$ Muthulekha Swamydas, ${ }^{1}$ Amanda L. Collar, ${ }^{1}$ Heidi H. Kong, ${ }^{7}$ Chyi-Chia Richard Lee, ${ }^{8}$ David Chascsa, ${ }^{9}$ Thomas Simcox, ${ }^{9}$ Angela Pham, ${ }^{1}$ Anamaria Bondici, ${ }^{1}$ Mukil Natarajan, ${ }^{1}$ Joseph Monsale, ${ }^{2}$ David E. Kleiner, ${ }^{7}$ Martha Quezado, ${ }^{7}$ Ilias Alevizos, ${ }^{10}$ Niki M. Moutsopoulos, ${ }^{11}$ Lynne Yockey, ${ }^{1,4}$ Cathleen Frein, ${ }^{5}$ Ariane Soldatos, ${ }^{12}$ Katherine R. Calvo, ${ }^{13}$ Jennifer Adjemian, ${ }^{14}$ Morgan N. Similuk, ${ }^{15}$ David M. Lang, ${ }^{16}$ Kelly D. Stone, ${ }^{17}$ Gulbu Uzel, ${ }^{4}$ Jeffrey B. Kopp, ${ }^{18}$ Rachel J. Bishop, ${ }^{19}$ Steven M. Holland, ${ }^{4}$ Kenneth N. Olivier, ${ }^{20}$ Thomas A. Fleisher, ${ }^{2}$ Theo Heller, ${ }^{9}$ Karen K. Winer, ${ }^{21}$ and Michail S. Lionakis

'Fungal Pathogenesis Unit, Laboratory of Clinical Infectious Diseases, National Institute of Allergy and Infectious Diseases (NIAID), ${ }^{2}$ Immunology Service, Department of Laboratory Medicine, NIH Clinical Center, ${ }^{3}$ Dental Clinical Research Core, National Institute of Dental and Craniofacial Research, and ${ }^{4}$ Immunopathogenesis Section, Laboratory of Clinical Infectious Diseases, NIAID, NIH, Bethesda, Maryland, USA. ${ }^{5}$ Clinical Research Directorate/Clinical Monitoring Research Program, Leidos Biomedical Research, Inc., National Cancer Institute (NCI) Campus at Frederick, Frederick, Maryland, USA. ${ }^{6}$ Biostatistics Research Branch, Division of Clinical Research, NIAID, Dermatology Branch, Center for Cancer Research, $\mathrm{NCl},{ }^{8}$ Laboratory of Pathology, Center for Cancer Research, NCl, ${ }^{9}$ Translational Hepatology Unit, Liver Diseases Branch, National Institute of Diabetes and Digestive and Kidney Diseases, ${ }^{10} \mathrm{~S}$ jögren's Syndrome and Salivary Gland Dysfunction Unit, and "Oral Immunity and Inflammation Unit, National Institute of Dental and Craniofacial Research, NIH, Bethesda Maryland, USA. ${ }^{12}$ Undiagnosed Diseases Program, Common Fund, NIH Office of the Director and National Human Cenome Research Institute, Bethesda, Maryland, USA. ${ }^{33}$ Hematology Section, Department of Laboratory Medicine, NIH Clinical Center, ${ }^{14}$ Epidemiology Unit, Laboratory of Clinical Infectious Diseases, ${ }^{15}$ Laboratory of Immunology, NIAID, ${ }^{16}$ Pediatric Consult Service, NIH Clinical Center, ${ }^{17}$ Laboratory of Allergic Diseases, NIAID, ${ }^{18}$ Kidney Diseases Branch, National Institute of Diabetes and Digestive and Kidney Diseases, ${ }^{19}$ Consult Services Section, National Eye Institute, ${ }^{20}$ Cardiovascular and Pulmonary Branch, National Heart, Lung, and Blood Institute, and ${ }^{21}$ Pediatric Growth and Nutrition Branch, Eunice Kennedy Shriver National Institute of Child Health and Human Development, NIH, Bethesda, Maryland, USA.

Authorship note: E.M.N. Ferre and S.R. Rose contributed equally to this work.

Conflict of interest: The authors have declared that no conflict of interest exists.

Submitted: May 27, 2016 Accepted: July 12, 2016 Published: August 18, 2016

Reference information: JCI Insight. 2016;1(13):e88782. doi:10.1172/jci.insight.88782.
Autoimmune polyendocrinopathy-candidiasis-ectodermal dystrophy (APECED) is a rare primary immunodeficiency disorder typically caused by homozygous AIRE mutations. It classically presents with chronic mucocutaneous candidiasis and autoimmunity that primarily targets endocrine tissues; hypoparathyroidism and adrenal insufficiency are most common. Developing any two of these classic triad manifestations establishes the diagnosis. Although widely recognized in Europe, where nonendocrine autoimmune manifestations are uncommon, APECED is less defined in patients from the Western Hemisphere. We enrolled 35 consecutive American APECED patients ( 33 from the US) in a prospective observational natural history study and systematically examined their genetic, clinical, autoantibody, and immunological characteristics. Most patients were compound heterozygous; the most common AIRE mutation was c.967_979del13. All but one patient had anti-IFN- $\omega$ autoantibodies, including 4 of 5 patients without biallelic AIRE mutations. Urticarial eruption, hepatitis, gastritis, intestinal dysfunction, pneumonitis, and Sjögren's-like syndrome, uncommon entities in European APECED cohorts, affected $40 \%-80 \%$ of American cases. Development of a classic diagnostic dyad was delayed at mean 7.38 years. Eighty percent of patients developed a median of 3 non-triad manifestations before a diagnostic dyad. Only $\mathbf{2 0} \%$ of patients had their first two manifestations among the classic triad. Urticarial eruption, intestinal dysfunction, and enamel hypoplasia were prominent among early manifestations. Patients exhibited expanded peripheral CD4 ${ }^{+} \mathrm{T}$ cells and $\mathrm{CD} 21^{10} \mathrm{CD} 38^{10}$ B lymphocytes. In summary, American APECED patients develop a diverse syndrome, with dramatic enrichment in organ-specific nonendocrine manifestations starting early in life, 


\section{Introduction}

APECED or autoimmune polyglandular syndrome type-1 (APS-1; OMIM 240300) is a monogenic disorder caused by biallelic mutations in autoimmune regulator (AIRE), a thymus-enriched transcription regulator that promotes central immune tolerance $(1,2)$. Recently, heterozygous dominant-negative AIRE mutations in the plant homeodomain 1 (PHD1) domain have also been described (3-5). APECED manifests with chronic mucocutaneous candidiasis (CMC) in $>80 \%-90 \%$ of patients and autoimmunity that primarily involves endocrine organs, including the parathyroids, adrenals, gonads, and thyroid; among these, hypoparathyroidism and adrenal insufficiency affect $>80 \%-90 \%$ of patients (6-8). Nonendocrine autoimmunity involving the liver, eye, kidney, and intestine has been reported in only a small minority $(\sim 5 \%-20 \%)$ of reported patients (6-12). Until now, diagnosis has relied upon the development of any two of the classic triad manifestations of CMC, hypoparathyroidism, and adrenal insufficiency, of which CMC is typically recognized earliest (6-12). Developing a diagnostic dyad typically prompts sequencing of AIRE and testing for anti-IFN- $\omega$ autoantibodies, which are highly prevalent among APECED patients (13).

APECED develops with high incidence $(1: 9,000-1: 25,000)$ in Finns, Sardinians, and Iranian Jews, and $>80 \%-90 \%$ of these patients carry the "signature" homozygous AIRE mutations c.769C >T (p.R257X), c.415C>T (p.R139X), and c.254A>G (p.Y85C), respectively $(6,12,14)$. APECED is also seen in Norway, Sweden, Slovenia, Slovakia, Russia, Great Britain, Italy, Ireland, and Poland, though it is associated with lower incidences $(\sim 1: 90,000-1: 200,000)$ and greater genetic diversity $(8-11,15-22)$. However, aside from small series, no systematic information has been reported about APECED in North America (23-25).

In order to fill this important gap, we comprehensively examined genetic, clinical, laboratory, autoantibody, and immunological characteristics of a large cohort of American APECED patients, enrolled consecutively for evaluation by a multidisciplinary team of specialists in a prospective observational natural history study. We found that American APECED has distinct genetic diversity, expanded $\mathrm{CD} 4^{+} \mathrm{T}$ and $\mathrm{CD} 21^{1 \circ} \mathrm{CD} 38^{\text {lo }} \mathrm{B}$ lymphocytes, and enriched organ-specific manifestations compared with those previously appreciated. We use these observations to suggest diagnostic criteria that would permit earlier diagnosis.

\section{Results}

Patient demographics. We enrolled 35 consecutive patients from 32 nonconsanguineous families with clinical and/or genetic diagnosis of APECED (inclusion criteria detailed in Methods) from North and South America (US, 33 [Supplemental Figure 1; supplemental material available online with this article; doi:10.1172/jci.insight.88782DS1]; Canada, 1; Colombia, 1). Dominant inheritance was not observed. Fourteen (40\%) were male, and twenty-one were female. The mean age was 20 years (range, 7-64 years); 16 (45.7\%) were children, with a mean age of 11.6 years. Thirty-one patients were of mixed European descent and four were Hispanic. Thirty-three patients self-reported $>1$ ethnic origin (mean, 2.76; range, 2-5), with Irish ancestry (51.4\%) being the most common, followed by German (40\%), British (31.4\%), French (20\%), Italian (17.1\%), and Scandinavian (17.1\%).

American APECED patients are genetically diverse, and approximately $15 \%$ do not carry biallelic AIRE mutations but have anti-IFN- $\omega$ autoantibodies. All exons, adjacent splicing sites, and highly conserved intronic, 5'UTR, and 3'UTR regions of the AIRE gene were sequenced. Biallelic mutations were identified in 29 (82.9\%) patients, whereas only a single mutation or no AIRE mutations were found in 2 (5.7\%) and 4 (11.4\%) patients, respectively. Among the 60 identified mutant alleles, c.967_979del13, seen in British, Norwegian, and Irish patients (38 alleles; 63.3\%), and c.769C>T, seen in Finns (12 alleles; 20\%), were most common (Supplemental Table 1) $(7,8,10,16,22)$ Among these alleles, 10 patients were homozygous for c.967_979del13, 9 were compound heterozygous for c.967_979del13 and c.769C>T, and 1 was homozygous for c.769C>T. Two patients were heterozygous for c.967_979del13 without another mutation detected. Seven patients were compound heterozygous for c.967_979del13 and one was compound heterozygous for c. $769 \mathrm{C}>\mathrm{T}$ in combination with another mutation. The 7 patients heterozygous for c.967_979del13 also 
A
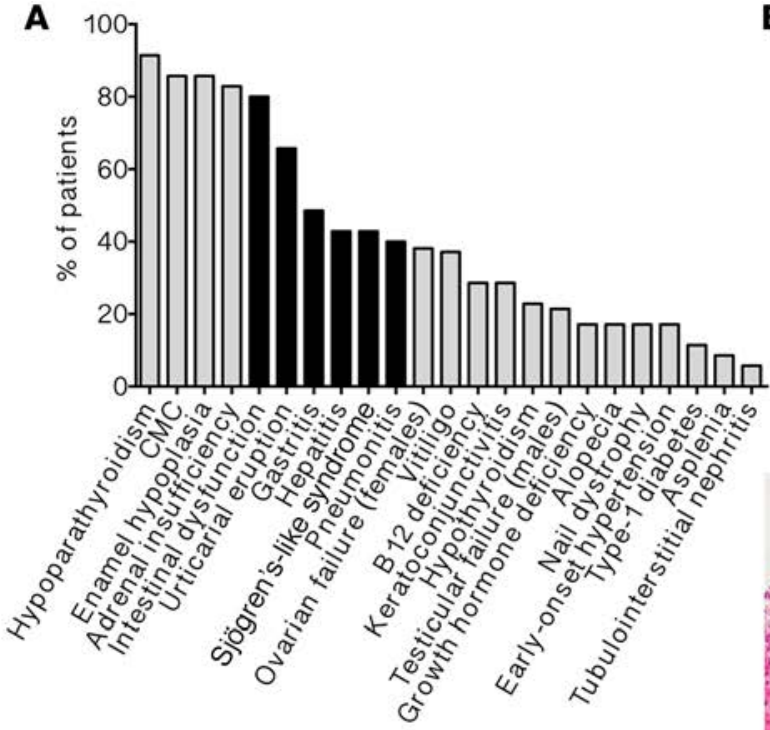

D

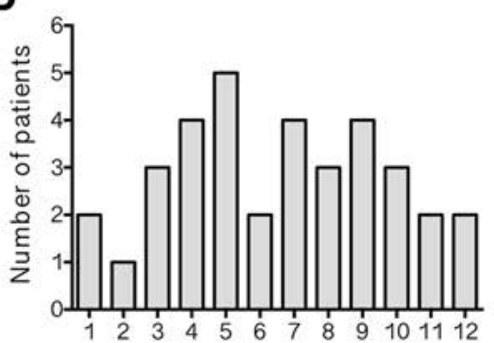

Number of non-endocrine manifestations per patient
B

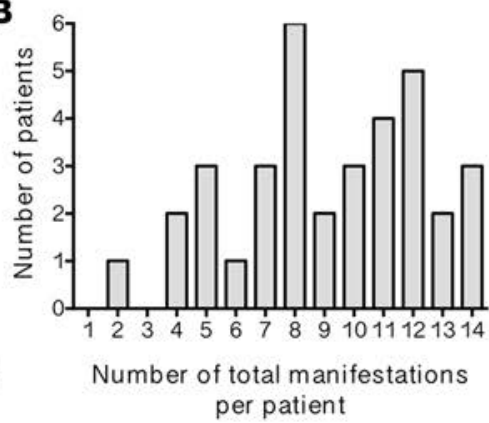

C

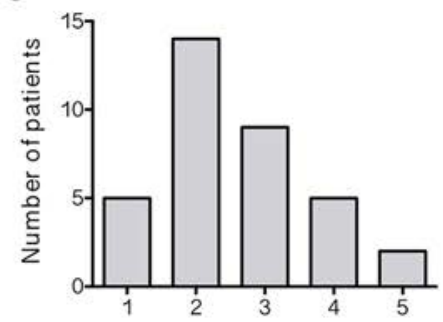

Number of endocrinopathies per patient
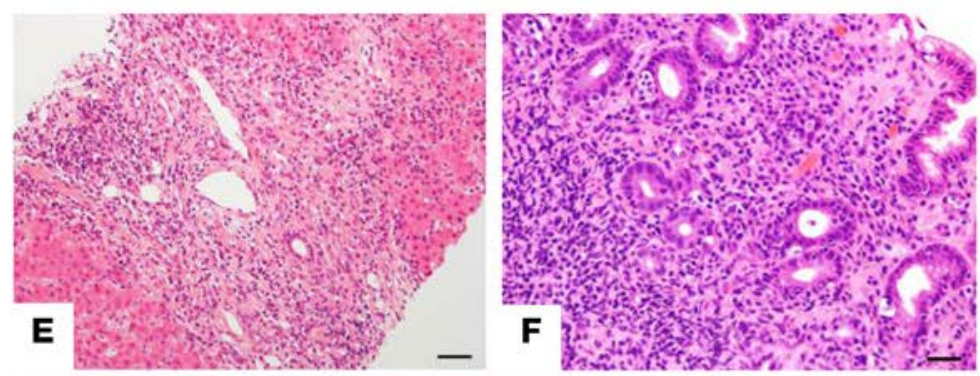

\section{E}

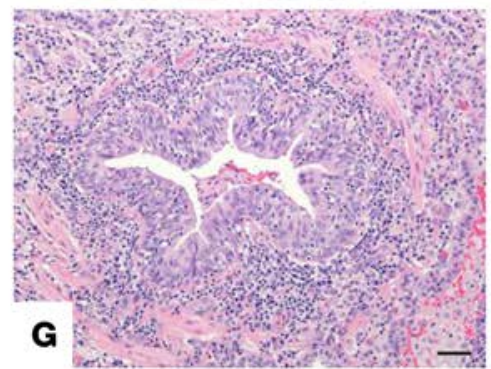

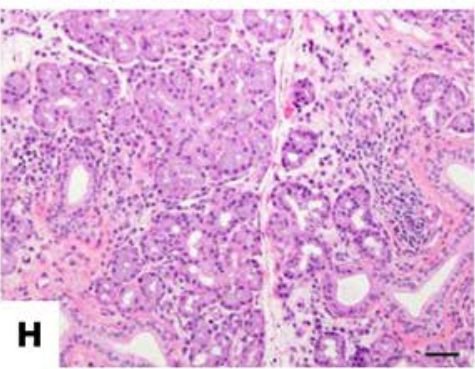

Figure 1. Enrichment of nonendocrine autoimmune manifestations in American autoimmune polyendocrinopathy-candidiasis-ectodermal dystrophy (APECED). (A) Prevalence of all disease manifestations in the 35 American APECED patients. The black bars represent the prominent hexad of nonendocrine organ-specific manifestations that is enriched in our cohort relative to all other reported experiences. (B-D) Distribution of all clinical manifestations (B), endocrinopathies (C), and nonendocrine manifestations (D) among the 35 American APECED patients. (E) Representative image from a liver biopsy of an APECED patient with autoimmune hepatitis showing severe chronic hepatitis with expansion of portal areas by inflammation and fibrosis. There is extensive interface hepatitis with numerous plasma cells infiltrating into the hepatic parenchyma (H\&E; scale bar: $50 \mu \mathrm{m}$; original magnification, $\times 200$ ). (F) Representative image from a stomach biopsy of an APECED patient with autoimmune gastritis showing chronic antral inflammation with lymphoplasmacytic infiltrates in the lamina propria and occasionally on glands (H\&E, scale bar: $50 \mu \mathrm{m}$; original magnification, $\times 200$ ). (G) Representative image from an open lung biopsy of an APECED patient with autoimmune pneumonitis showing chronic bronchiolitis with lymphocytic infiltration within and around the bronchiolar mucosa and lymphoid aggregates in the interstitium nearby (H\&E, scale bar: $50 \mu \mathrm{m}$; original magnification, $\times 200)$. (H) Representative image from a minor salivary gland biopsy in an APECED patient with Sjögren's-like syndrome showing lymphocytic and plasma cell infiltration in and around the ducts of the gland (H\&E; scale bar: $50 \mu \mathrm{m}$; original magnification, $\times 200$ ).

carried c.190_226del37 $(n=3)$, c.1249_1250insC $(n=2)$, c.1616C $>$ T $(n=1)$, or c.522_523ins13 $(n=1)$, whereas 1 patient was compound heterozygous for c.769C>T and c.789_789delC. One patient was homozygous for c.328delC.

We next examined whether the 6 patients in whom sequencing did not reveal biallelic mutations had AIRE deletions, as previously described (26). We employed comparative genomic hybridization (CGH) and found that 1 patient heterozygous for c.967_979del13 also had a 1,781-bp deletion spanning 7 bp before exon 1 through $20 \mathrm{bp}$ after exon 4 on the other allele; no AIRE deletions/duplications were detected in the remaining 5 patients. Collectively, among the 30 patients with biallelic AIRE mutations/deletions, the majority $(19 ; 63.3 \%)$ were compound heterozygous for different alleles or deletions, while only 11 (36.7\%) were homozygous. Taken together, American APECED patients exhibit more genetic diversity than European patients, as previously suggested in a small number of patients (27), and approximately $15 \%$ of patients with clinical APECED do not carry biallelic AIRE mutations. These findings suggest that variants distinct from the conventionally recognized AIRE locus may contribute to APECED in 
Table 1. Prevalence of disease manifestations by age at presentation in American autoimmune polyendocrinopathy-candidiasis-ectodermal dystrophy (APECED) patients

\begin{tabular}{|c|c|c|c|c|c|c|c|c|c|c|c|}
\hline & \multicolumn{10}{|c|}{ Age (yr) } & \multirow{2}{*}{$\begin{array}{c}\text { Age range } \\
\text { (median, mean) } \\
\text { (yr) }\end{array}$} \\
\hline & 1 & 2 & 5 & 10 & 15 & 20 & 30 & 40 & 50 & $60+$ & \\
\hline \multicolumn{12}{|l|}{ APECED clinical diagnostic criteria } \\
\hline Classic triad & $0 \%$ & $3 \%$ & $11 \%$ & $37 \%$ & $49 \%$ & $54 \%$ & $60 \%$ & $63 \%$ & $63 \%$ & $63 \%$ & $3-34(10,12.1)$ \\
\hline $\begin{array}{l}\text { Diagnostic dyad within expanded } \\
\text { criteria }\end{array}$ & $40 \%$ & $57 \%$ & $77 \%$ & $94 \%$ & $100 \%$ & $100 \%$ & $100 \%$ & $100 \%$ & $100 \%$ & $100 \%$ & $0.25-14(2,3.5)$ \\
\hline $\begin{array}{l}\text { Chronic mucocutaneous } \\
\text { candidiasis }\end{array}$ & $51 \%$ & $57 \%$ & $63 \%$ & $74 \%$ & $80 \%$ & $80 \%$ & $86 \%$ & $86 \%$ & $86 \%$ & $86 \%$ & $0.5-26(1,4.4)$ \\
\hline Enamel hypoplasia & $0 \%$ & $11 \%$ & $37 \%$ & $66 \%$ & $80 \%$ & $83 \%$ & $86 \%$ & $86 \%$ & $86 \%$ & $86 \%$ & $2-21(6,7.7)$ \\
\hline \multicolumn{12}{|l|}{ Endocrine manifestations } \\
\hline Hypoparathyroidism & $6 \%$ & $29 \%$ & $57 \%$ & $83 \%$ & $86 \%$ & $89 \%$ & $91 \%$ & $91 \%$ & $91 \%$ & $91 \%$ & $0.5-29(5,5.9)$ \\
\hline Adrenal insufficiency & $0 \%$ & $6 \%$ & $23 \%$ & $54 \%$ & $71 \%$ & $77 \%$ & $80 \%$ & $83 \%$ & $83 \%$ & $83 \%$ & $2-34(8,9.9)$ \\
\hline Testicular failure ${ }^{\mathrm{B}}$ & $0 \%$ & $0 \%$ & $0 \%$ & $0 \%$ & $7 \%$ & $14 \%$ & $14 \%$ & $14 \%$ & $14 \%$ & $21 \%$ & $13-52(19,28)$ \\
\hline \multicolumn{12}{|l|}{ Gastrointestinal manifestations } \\
\hline Intestinal dysfunction & $26 \%$ & $40 \%$ & $43 \%$ & $51 \%$ & $77 \%$ & $80 \%$ & $80 \%$ & $80 \%$ & $80 \%$ & $80 \%$ & $1-16(3,5.1)$ \\
\hline Hepatitis & $0 \%$ & $9 \%$ & $11 \%$ & $23 \%$ & $31 \%$ & $40 \%$ & $40 \%$ & $43 \%$ & $43 \%$ & $43 \%$ & $2-31(9,10.5)$ \\
\hline Gastritis & $0 \%$ & $6 \%$ & $11 \%$ & $23 \%$ & $37 \%$ & $37 \%$ & $40 \%$ & $46 \%$ & $46 \%$ & $49 \%$ & $2-54(11,15.1)$ \\
\hline B12 deficiency & $0 \%$ & $0 \%$ & $3 \%$ & $9 \%$ & $11 \%$ & $23 \%$ & $26 \%$ & $29 \%$ & $29 \%$ & $29 \%$ & $2-31(17,16.5)$ \\
\hline \multicolumn{12}{|l|}{ Skin/nail manifestations } \\
\hline Urticarial eruption & $49 \%$ & $60 \%$ & $63 \%$ & $63 \%$ & $63 \%$ & $66 \%$ & $66 \%$ & $66 \%$ & $66 \%$ & $66 \%$ & $0.25-17(1,1.6)$ \\
\hline Alopecia & $0 \%$ & $3 \%$ & $6 \%$ & $11 \%$ & $17 \%$ & $17 \%$ & $17 \%$ & $17 \%$ & $17 \%$ & $17 \%$ & $2-15(8,8)$ \\
\hline Vitiligo & $0 \%$ & $3 \%$ & $9 \%$ & $17 \%$ & $23 \%$ & $31 \%$ & $31 \%$ & $34 \%$ & $34 \%$ & $37 \%$ & $2-63(11,15.5)$ \\
\hline
\end{tabular}

a sizable proportion of American patients. Importantly, because genetic confirmation of the clinical diagnosis in these patients would be missed by sequencing, we examined whether anti-IFN- $\omega$ autoantibodies were present in our cohort. We found that 34 of 35 patients (97.1\%) were positive, 100\% (30 of 30 patients) and $80 \%$ ( 4 of 5 patients) of those with or without detectable biallelic AIRE mutations, respectively (Supplemental Figure 2).

American APECED is enriched for nonendocrine manifestations. We admitted all patients to the NIH and obtained detailed medical histories; then, comprehensive clinical, laboratory, and radiographic evaluations were performed by a multidisciplinary team of specialists with an aim to systematically record all clinical manifestations in our patients and identify common and distinct features relative to other APECED cohorts. We also measured autoantibodies that have been associated with specific disease manifestations in reported cohorts and examined whether they correlated with the time to development of the corresponding clinical manifestations in our patients. We found a dramatic expansion of disease manifestations, with 23 clinical entities seen in varying frequencies (5.7\%-91.4\%; Figure 1A and Table 1), with a median of 9 
manifestations per patient (mean, 9.1; range, 2-14; Figure 1B). We also found a correlation between the c. $769 \mathrm{C}>\mathrm{T}$ allele and the time to development of type 1 diabetes and nail dystrophy (Supplemental Figure 3 ), which requires validation in future cohorts.

The observed spectrum and frequency of endocrine manifestations were similar to those in reported cohorts (6-22). Patients developed a median of 2 endocrinopathies (mean, 2.6; range, 1-5; Figure 1C), with hypoparathyroidism and adrenal insufficiency being the most common ( $85 \%-90 \%)$, followed by ovarian failure $(38.1 \%$ of females), hypothyroidism (22.9\%), testicular failure (21.4\% of males), growth hormone (GH) deficiency (17.1\%), and type 1 diabetes (11.4\%). Among endocrinopathies, autoantibodies against islet antigen-2 (IA-2), but not glutamic acid decarboxylase 65 (GAD65), were significantly associated with the time to development of diabetes, while side-chain cleavage enzyme (SCC) and/or 21-hydroxylase autoantibodies showed a trend toward earlier development of adrenal insufficiency (Supplemental Table 2 and Supplemental Figures 4 and 5) (28, 29).

In contrast to endocrinopathies, the spectrum and frequency of nonendocrine manifestations were distinct in our patients: they developed a median of 7 such manifestations (mean, 6.6; range 1-12; Figure 1D), with a median of 3 nonendocrinopathies (mean, 3.23; range, 1-9) before their first endocrinopathy. Some of the same nonendocrine entities that manifested in prior reports manifested in our cohort (6-12, 15-22): CMC was the "signature" infection, occurring in 30 (86\%); oral thrush developed in all, followed by vulvovaginal (52.4\% of females), esophageal (51.4\%), nail (34.3\%), and cutaneous candidiasis (17.1\%). No oral or esophageal squamous cell carcinomas were seen. Autoantibodies against IL-22 and IL-17F, but not IL-17A, were significantly associated with the time to development of CMC (Supplemental Table 2 and Supplemental Figure 6) $(30,31)$. Additional diseases with comparable frequencies relative to reported cohorts included enamel hypoplasia (85.7\%), vitiligo (37.1\%), keratoconjunctivitis $(28.6 \%)$, nail dystrophy (17.1\%), early-onset hypertension (17.1\%), asplenia (8.6\%), and tubulointerstitial nephritis (5.7\%), while alopecia (17.1\%) appeared less often (6-22).

Remarkably, organ-specific nonendocrine manifestations were highly enriched in our cohort. Urticarial eruption was seen in $66 \%$ of patients, as opposed to its absence or sporadic occurrence in previous reports (6-22). Hepatitis and intestinal dysfunction were seen in $42.9 \%$ and $80 \%$ of patients, respectively, rates substantially higher than in previous reports $(\sim 5 \%-20 \%)(6-22)$. Autoantibodies against tryptophan hydroxylase (TPH), but not GAD65, were significantly associated with the time to development of intestinal dysfunction (Supplemental Table 2 and Supplemental Figures 4 and 5) (32). Moreover, biopsy-proven gastritis was seen in 17 patients (48.6\%); 10 patients (28.6\%) had B12 deficiency associated with positive intrinsic-factor antibody, which was significantly associated with the time to development of B12 deficiency (Supplemental Table 2 and Supplemental Figures 4 and 5).

Along with these cutaneous and intra-abdominal complications, a substantial proportion of patients developed two additional nonendocrine entities that have only sporadically been reported in APECED. Specifically, $42.9 \%$ of patients developed a Sjögren's-like syndrome without extractable nuclear antigen (ENA) autoantibodies, suggesting that salivary inflammation in APECED is distinct from that in idiopathic Sjögren's syndrome. Furthermore, $40 \%$ of patients developed pneumonitis. Autoantibodies against bactericidal/permeability-increasing fold-containing B1 (BPIFB1) and the potassium channel regulator KCNRG were significantly associated with the time to development of pneumonitis. BPIFB1 was highly sensitive, whereas KCNRG lacked sensitivity but was 100\% specific (Supplemental Table 2 and Supplemental Figures 4 and 5) $(33,34)$. A single 14-year-old patient in our cohort died (mortality, 2.9\%) from pneumonitis-related respiratory failure, attesting to its life-threatening potential. Lymphocytic infiltration was seen in the liver, stomach, lungs, and salivary glands of patients with corresponding tissue autoimmunity (Figure 1, E-H). Collectively, our patients manifested a high prevalence of a hexad of organ-specific nonendocrine diseases (i.e., urticarial eruption, hepatitis, intestinal dysfunction, gastritis, Sjogren's-like syndrome, pneumonitis), which were not previously appreciated as common in non-American cohorts; specifically, 91.4\%, 80\%, 65.7\%, 42.9\%, 25.7\% and $14.3 \%$ of all patients developed 1,2, 3, 4, 5 or all 6 of the nonendocrine diseases among this hexad, respectively (Supplemental Table 3).

We next wondered whether the distinctive genotypic signature of our patient cohort (Supplemental Table 1) might play an important role in the enrichment of these 6 nonendocrine manifestations in American APECED. To assess for genotype-phenotype correlations, we compared the time to development of urticarial eruption, hepatitis, intestinal dysfunction, gastritis, Sjögren's-like syndrome, and pneumonitis among the 27 patients with biallelic AIRE mutations that include c.967_979del13 in het- 
A

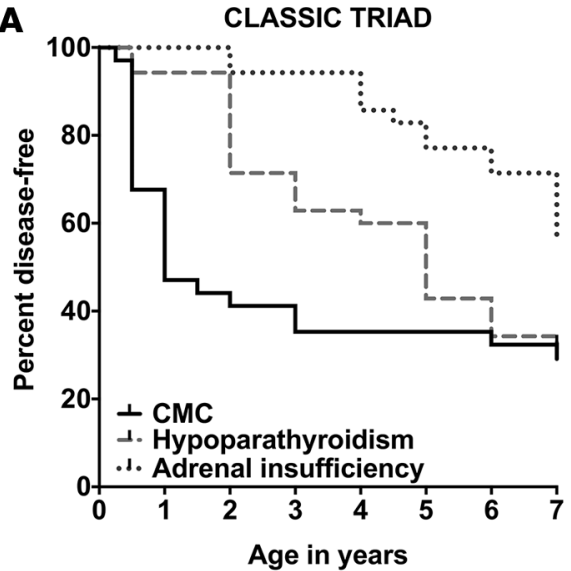

C

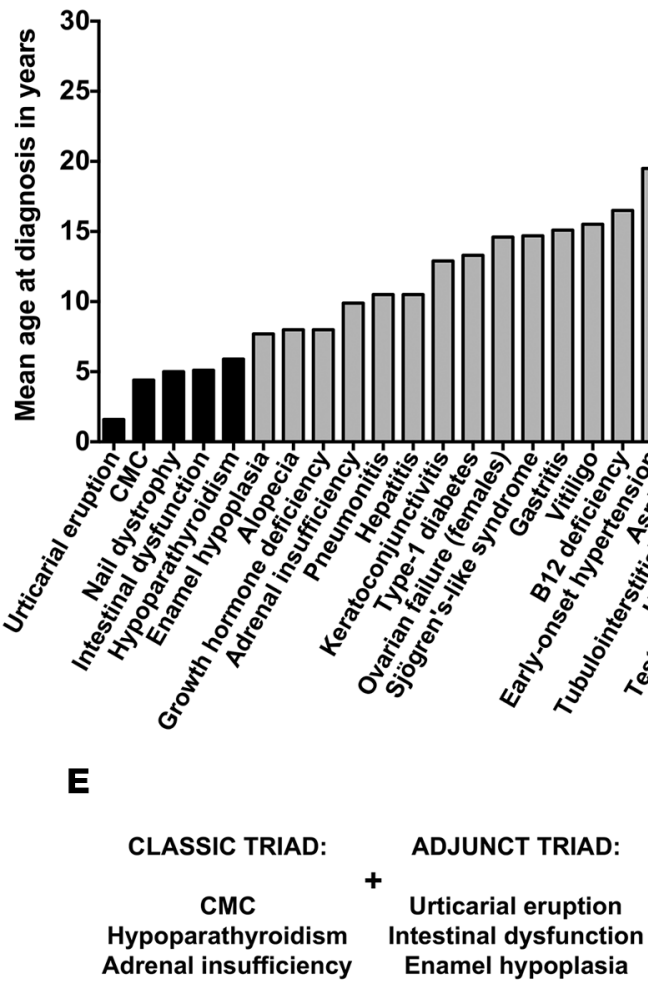

Any 2 manifestations within the combined classic and adjunct triads

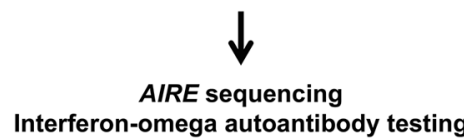

B

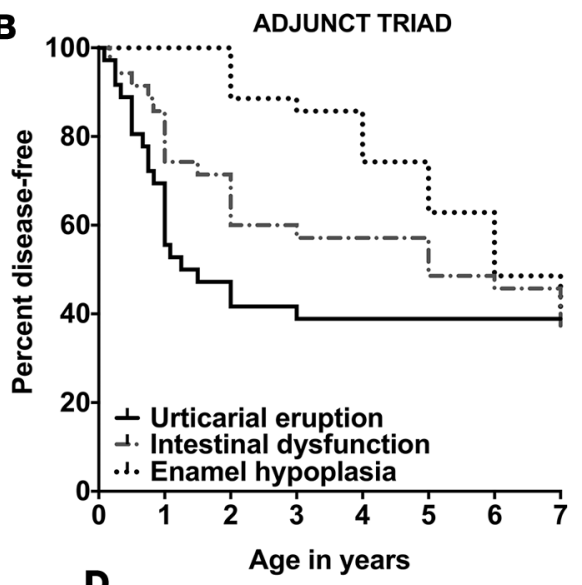

Any 2 manifestations within:

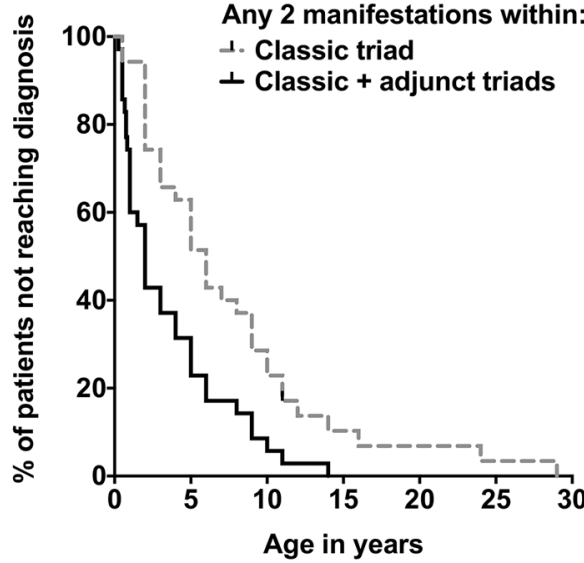

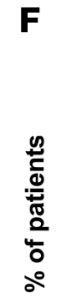

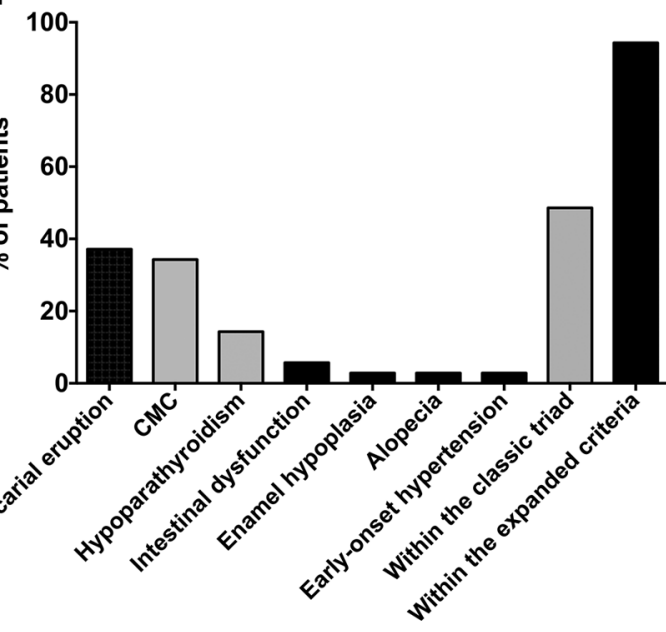

First APECED manifestation

Figure 2. Early manifestations and redefined diagnostic criteria in American autoimmune polyendocrinopathy-candidiasis-ectodermal dystrophy (APECED). (A and B) Prevalence by age of the classic triad manifestations (A) and the adjunct triad of urticarial eruption, intestinal dysfunction, and enamel hypoplasia (B) within the first 7 years of life, before the mean age at which a diagnostic dyad was reached in the 35 American APECED patients. (C) Mean age at diagnosis of all clinical manifestations among the APECED patients who developed the corresponding disease components. Black bars denote the clinical manifestations with a mean age of diagnosis within the first 7 years of life, before the mean age at which a classic diagnostic dyad is reached. (D) Age of reaching diagnosis based on development of any 2 classic triad manifestations (current diagnostic criteria) versus reaching the diagnosis based on development of any 2 manifestations within the combined classic triad and adjunct triad of urticarial eruption, intestinal dysfunction, and enamel hypoplasia (expanded diagnostic criteria). (E) Redefined expanded diagnostic criteria and diagnostic algorithm aimed at promoting earlier diagnosis of American APECED patients. (F) Distribution of the initial manifestation in the 35 American APECED patients. Gray bars denote manifestations within the current classic diagnostic triad. 

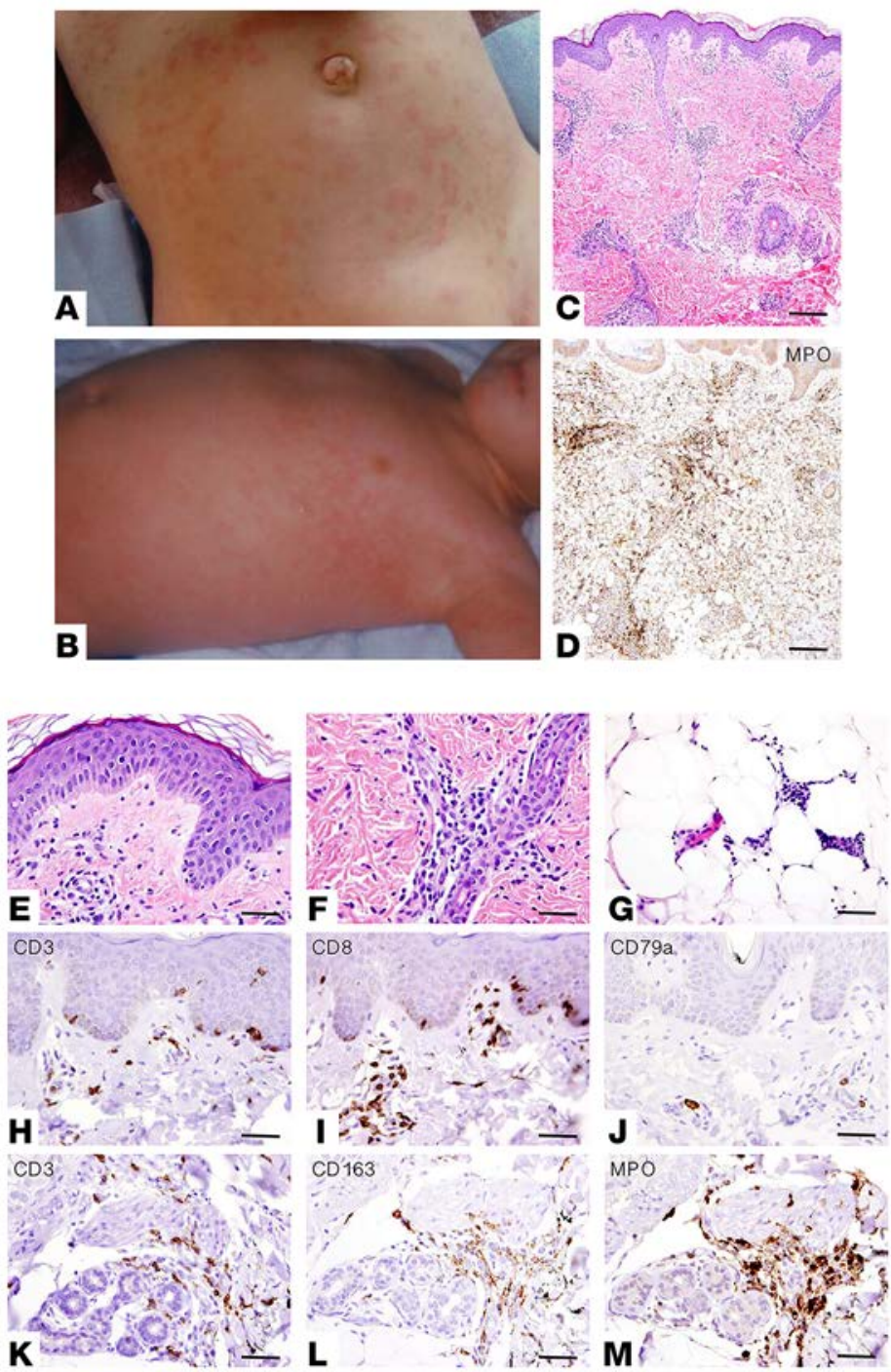

Figure 3. Clinical and histological presentation of urticarial eruption in American children with autoimmune polyendocrinopathy-candidiasis-ectodermal dystrophy (APECED). (A and $\mathbf{B}$ ) Representative images showing erythematous urticarial eruption on the torsos of two children with APECED. (C) Histologic examination of skin biopsies reveals perivascular and periadnexal inflammation in the superficial and deep dermis with pallor of the papillary dermis (H\&E; scale bar: $2 \mathrm{~mm}$; original magnification, $\times 40)(D)$ Myeloperoxidase (MPO) immunohistochemical staining highlights the presence of neutrophils and karyorrhectic debris derived from neutrophils (scale bar: $2 \mathrm{~mm}$; original magnification, $\times 40)$. (E) High-magnification view of the epidermis and superficial dermis revealing focal interface vacuolar changes along the epidermal basement membrane zone, with thickened and irregular basement membrane and superficial perivascular inflammation, with karyorrhectic debris in the dermis (H\&E; scale bar: $200 \mu \mathrm{m}$; original magnification, $\times 200$ ). (F) High-magnification view of the reticular dermis revealing perivascular and interstitial inflammation predominantly composed of mononuclear cells with karyorrhectic debris, while mature neutrophils are infrequent (H\&E; scale bar: $200 \mu \mathrm{m}$; original magnification, $\times 200)$. (G) High-magnification view of the subcutaneous adipose tissue revealing inflammation predominantly composed of mononuclear cells with karyorrhectic debris ( $\mathrm{H} \& \mathrm{E}$; scale bar: $300 \mu \mathrm{m}$; original magnification, $\times 200)$. (H-J) Immunohistochemical staining with lymphocyte markers (CD3, H; CD8, I; CD79a, J; CD4, not shown) revealing lymphocytic exocytosis with $\mathrm{CD}^{+}$and $\mathrm{CD} 8^{+} \mathrm{T}$ lymphocytes lining up along the base of epidermis and occasional cells within the spinous layers; CD79a $B$ lymphocytes are rarely seen in a perivascular distribution (scale bar: $200 \mu \mathrm{m}$; original magnification, $\times 400$ ). (K-M) Immunohistochemical staining with lymphocyte markers (CD3, K), macrophage/monocyte/histiocyte markers (CD163, L; CD68, not shown), and neutrophil/myeloid lineage markers (MPO, M) revealing periadnexal/perieccrine inflammation composed of abundant neutrophils/myeloid cells with karyorrhexis and scattered lymphocytes and monocytes/macrophages/histiocytes (scale bar: $200 \mu \mathrm{m}$; original magnification, $\times 400$ ).

erozygosity ( $n=17$ ) or homozygosity $(n=10)$ (group A), the 3 APECED patients with biallelic AIRE mutations other than c.967_979del13 (group B), and the 5 APECED patients without biallelic AIRE mutations (group C). We found a significant association between the time to development of urticarial eruption or hepatitis and carrying biallelic AIRE mutations other than c.967_979del13 (group B) relative to not carrying biallelic AIRE mutations (group C); no genotype-phenotype correlations were observed for the other 4 organ-specific nonendocrine manifestations (Supplemental Figure 7). Among all the other clinical manifestations, we found a significant negative association between the time to development of testicular failure and carrying biallelic AIRE mutations that include c.967_979del13 in heterozygosity or homozygosity (group A) relative to genotypes in groups B or C; no other genotype-phenotype correlations were observed (Supplemental Figure 7). Given the small numbers of patients in groups B and $C$, future studies using a larger cohort of patients will be required to conclusively determine whether any genotype-phenotype correlations may account, at least in part, for the enriched nonendocrine manifestations of American APECED patients.

Urticarial eruption, intestinal dysfunction, and enamel hypoplasia are common early APECED manifestations. The clinical diagnosis of APECED currently relies on developing any two manifestations within the classic triad of CMC, hypoparathyroidism, and adrenal insufficiency (6-9). The classic triad was seen in $22(62.9 \%)$ patients, whereas a diagnostic dyad was seen in all patients, with the exception of an 11-yearold girl homozygous for c.967_979del13, who had isolated hypoparathyroidism among the classic triad manifestations (Table 1). Although a diagnostic dyad was highly prevalent, we observed that its devel- 
A

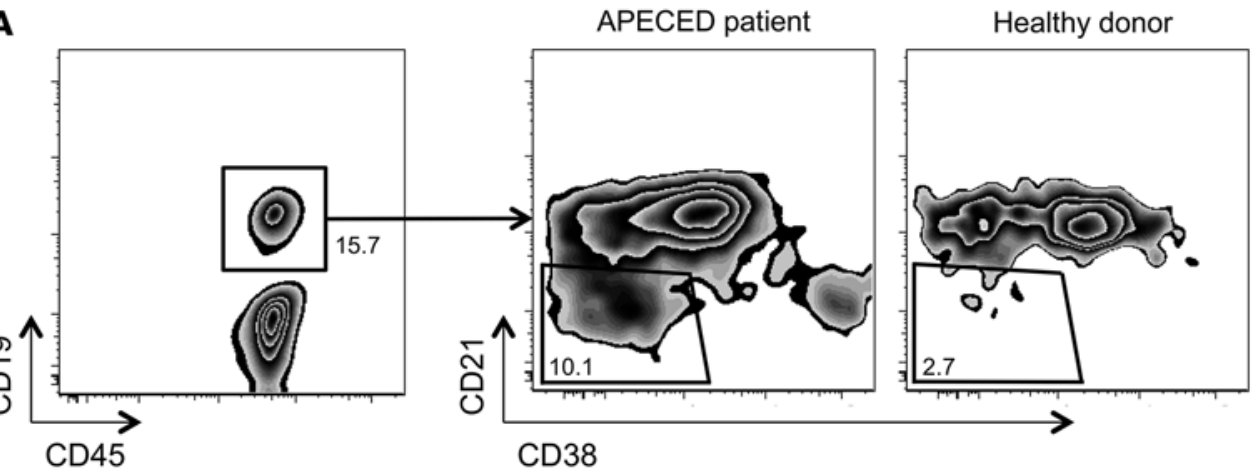

B

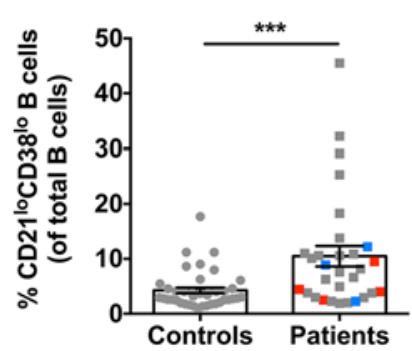

E

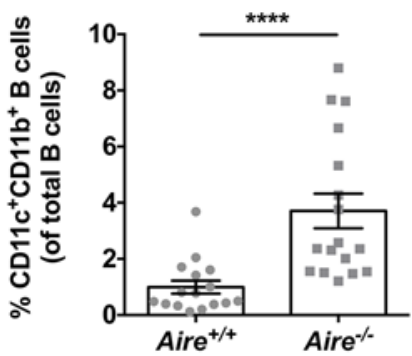

C

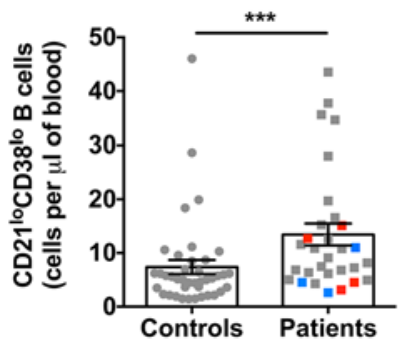

$\mathbf{F}$

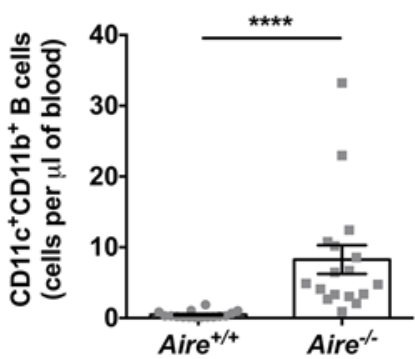

D

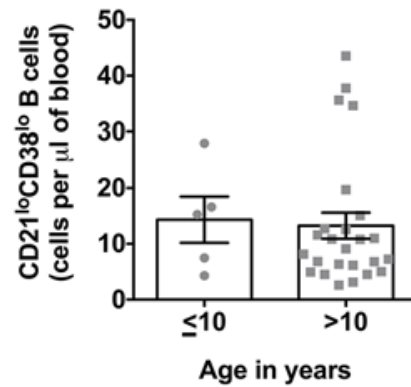

Figure 4. Expansion of $\mathrm{CD} 21^{10} \mathrm{CD} 38^{10} \mathrm{~B}$ cells in the peripheral blood of autoimmune polyendocrinopathy-candidiasis-ectodermal dystrophy (APECED) patients and Aire $/-$ mice. (A) Gating strategy and representative FACS plots of $\mathrm{CD} 21^{1{ }^{\circ} \mathrm{CD} 38^{\circ} \mathrm{B}} \mathrm{B}$ cells in peripheral blood. After initial FSC/SCC gating of peripheral blood mononuclear cells (not shown), $C D 19+B$ cells were selected (left) and $C D 21^{10} \mathrm{CD} 38^{10} \mathrm{~B}$ cells were delineated and quantified. Representative FACS plots of $C D 21^{10} \mathrm{CD} 38^{10} \mathrm{~B}$ cells from an APECED patient (middle) and a healthy donor (right) showing enrichment of these cells in patient peripheral blood. (B and $\mathbf{C}$ ) Summary data for the percentage of CD21 $21^{\circ} \mathrm{CD} 38^{10} \mathrm{~B}$ cells within total $C D 19+B$ cells $\left(B,{ }^{* *} P=0.0004\right.$, Mann-Whitney test) and for the absolute number of $\operatorname{CD} 21^{10} \mathrm{CD} 38^{10} \mathrm{~B}$ cells per $\mu$ l of human blood (C, ${ }^{* *} P=0.0007$, Mann-Whitney test). APECED patients, $n=30$; healthy donors, $n=40$. The 23 gray squares correspond to the results obtained in the 23 APECED patients with biallelic AIRE mutations that include c.967_979del13 in heterozygosity or homozygosity (group A), the 3 blue squares correspond to the results obtained in the 3 APECED patients with biallelic AIRE mutations other than c.967_979del13 (group B), and the 4 red squares correspond to the results obtained in the 4 APECED patients without biallelic AIRE mutations (group $\mathrm{C}$ ). The percentage and absolute number of CD21 ${ }^{10} \mathrm{CD} 38^{10} \mathrm{~B}$ cells did not significantly differ between patient groups $\mathrm{A}, \mathrm{B}$, and C (unpaired $t$ test). (D) Summary data for the absolute number of $\operatorname{CD} 21^{10} \mathrm{CD} 38^{\circ} \mathrm{B}$ cells per $\mu$ l of blood of APECED patients that are younger $(n=5)$ or older $(n=25)$ than 10 years. (E and F) Summary data for the percentage of $C D 11 c^{+} C D 11 b^{+} B$ cells within total CD19+ $B$ cells (E, ${ }^{* * *} P<0.0001$, Mann-Whitney test) and for the absolute number of CD11c ${ }^{+} \mathrm{CD}_{11} b^{+} B$ cells per $\mu$ l of mouse blood (F, ${ }^{* * *} P<0.0001$, Mann-Whitney test). Aire $^{+/+}$mice, $n=16$; Aire $^{-/-}$mice, $n=17 ; 4$ independent experiments. All quantitative data represent mean $\pm \mathrm{SEM}$.

opment occurred at a mean age of 7.38 years (range, 0.5-29 years). Before achieving a diagnostic dyad, most patients had developed other disease components, which had not sparked suspicion for APECED. Therefore, we reasoned that systematic documentation of all clinical manifestations and their ages of onset might identify early disease aspects that could complement the existing diagnostic criteria and foster more timely diagnosis. 
Unexpectedly, we found that only 7 (20\%) patients developed a classic dyad of diagnostic criteria, CMC and hypoparathyroidism, as part of their first two manifestations. In contrast, 27 patients developed a median of 3 manifestations (mean, 3; range, 1-8) before they met a diagnostic dyad. The remaining patient was an 11-year-old girl homozygous for c.967_979del13 who had not yet developed a dyad but already had 3 non-triad manifestations. Thus, $80 \%, 60 \%, 46 \%$, and $29 \%$ of our 35 patients did not meet the classic diagnostic criteria by their second, third, fourth, or fifth consecutive clinical manifestations, respectively. Therefore, multiple non-triad manifestations occur in APECED patients well before reaching a classic diagnostic dyad. These data suggested that the classic criteria were permitting unnecessary delay in APECED diagnosis and causing missed opportunities for therapeutic and prophylactic intervention.

We analyzed the manifestations appearing before development of a classic dyad in the 28 patients for whom the diagnostic criteria did not develop as part of their first two manifestations. Remarkably, we observed varying frequencies $(3.6 \%-78.6 \%)$ of 15 manifestations occurring before the development of diagnostic criteria in these patients (Supplemental Table 4). The three most common were urticarial eruption (78.6\%), intestinal dysfunction (53.6\%; Supplemental Table 5), and enamel hypoplasia (42.9\%), which were seen either alone or in combination in $25(\sim 90 \%)$ of these 28 patients well before the conventional diagnostic criteria were met (Figure 2, A-C). Sjögren's-like syndrome, pneumonitis, keratoconjunctivitis, hepatitis, and nail dystrophy were seen in 7 (25\%), 7 (25\%), 4 (14.3\%), 4 (14.3\%) and 4 (14.3\%) patients, respectively (Supplemental Table 4).

If urticarial eruption, intestinal dysfunction, and enamel hypoplasia had been added to the classic triad manifestations, the diagnosis would have been reached with the first two consecutive manifestations in $71.4 \%(n=25)$ instead of only $20 \%(n=7)$ of patients (Supplemental Table 6). Importantly, the mean age for reaching a diagnosis based on any 2 manifestations within the combined classic triad and an adjunct triad of urticarial eruption, intestinal dysfunction, and enamel hypoplasia (expanded diagnostic criteria) would have been 3.54 years (median, 2 years), almost 4 years earlier than the current 7.38 years (median, 6 years) at which the current diagnostic dyad within the classic triad criteria is reached (Figure 2, D and E, and Table 1). We then asked whether establishing an earlier diagnosis by approximately 4 years based on the expanded diagnostic criteria would have prevented episodes of life-threatening hypocalcemic seizures and adrenal crises, which are currently principal presentations of undiagnosed APECED patients. Indeed, among the 28 patients in whom the classic diagnostic criteria did not develop as part of their first two manifestations, 13 patients (46.4\%) would have been diagnosed with APECED by reaching a diagnostic dyad within the expanded diagnostic criteria but not within the classic triad before they were hospitalized with hypocalcemic seizures $(n=11 ; 39.3 \%)$ or combined hypocalcemic seizures and adrenal crises $(n=2 ; 7.1 \%)$. These data indicate that earlier diagnosis by the proposed expanded diagnostic criteria may have significant implications for prevention of life-threatening endocrine complications in clinical practice.

A characteristic urticarial eruption is the most common initial manifestation in American APECED. We focused on the urticarial eruption because it is a poorly recognized early manifestation in APECED and it was the most common initial manifestation among all patients, along with $\mathrm{CMC}(37.1 \%$, Figure $2 \mathrm{~F})$, with a mean onset at 1.6 years (Figure 2C). We systematically collected information on patients' urticarial eruptions and found that urticarial eruptions presented as flat or more commonly as a raised erythematous widespread eruption presenting between 3 and 36 months, with the exception of a single patient who developed classical urticaria at 17 years. Fever accompanied urticarial eruptions in 5 patients. The rash was mostly nonpruritic (90.9\%) and nonblanching $(72.7 \%)$ and often started on the torso, with spread to the face and extremities, while sparing the palms and soles (Figure 3, A and B, and Supplemental Table 7). It resolved after a mean of 146.8 days, but in $72.7 \%$ of the cases, it recurred with identical features.

Histologic examination of skin biopsies obtained from three 11- to 15-month-old APECED children revealed mild-to-moderate superficial and deep dermal perivascular inflammation, without vasculitic features of thrombi and fibrin deposition. We observed characteristic patchy mixed myeloid and lymphoid infiltrates consisting of $\mathrm{T}$ lymphocytes, myeloperoxidase ${ }^{+}\left(\mathrm{MPO}^{+}\right)$neutrophils with associated karyorrhexis, $\mathrm{CD}_{163^{+}}$monocytes/macrophages, and occasional plasma cells and eosinophils. We also observed mild interface vacuolar changes at the dermal-epidermal junction, with lymphocytic exocytosis and foci of panniculitis in a lobular pattern (Figure 3, C-M, and Supplemental Table 8). Taken together, urticarial eruption was the most common initial manifestation in American APECED patients, and recognition of its characteristic clinical and histological presentation could facilitate early diagnosis. 
$C D 4^{+} T$ and $C D 21^{\text {to } C D} 38^{\text {to }} B$ lymphocytes are expanded in APECED. Immunophenotyping of peripheral blood $\mathrm{T}$ lymphocytes has previously been reported in small numbers of APECED patients (35-37). We systematically analyzed all peripheral blood lymphocyte subpopulations in the patients relative to healthy donors to identify characteristic immunological features associated with APECED. The patients exhibited increased $\mathrm{T}$ lymphocytes due to significantly expanded $\mathrm{CD} 4^{+} \mathrm{T}$ cells, leading to increased CD4/CD8 ratios (Supplemental Tables 9 and 10), consistent with prior reports (35-37). The $\mathrm{CD}^{+} \mathrm{T}$ cell enrichment was seen in naive, but not central memory, effector memory or effector cell subsets. The patients had significantly decreased NK cells and normal NKT cells (Supplemental Tables 9 and 10).

Patients had normal total B cells and normal representation of immature, transitional, and memory B cell subsets, while plasmablasts were greater in patients' blood (Supplemental Tables 9 and 10). Because APECED is an autoimmune disorder associated with production of a broad panel of autoantibodies, we focused on the autoreactive $\mathrm{CD} 21^{\text {lo }} \mathrm{CD} 38^{\text {lo }} \mathrm{B}$ cell subset (38), which is enriched in patients with common variable immunodeficiency-associated autoimmune cytopenias, in patients with cytotoxic $\mathrm{T}$ lymphocyteassociated protein 4 (CTLA4) haploinsufficiency-associated immune dysregulation, and in some patients with systemic lupus erythematosus and rheumatoid arthritis (39-42). We found a significant expansion of $\mathrm{CD} 21^{10} \mathrm{CD} 38^{\text {lo }} \mathrm{B}$ cells in APECED patients from an early age, the extent of which did not correlate with specific AIRE mutations (Figure 4, A-D, and Supplemental Tables 9 and 10). To verify the AIRE deficiency-dependent enrichment in these cells, we examined 5- to 6-week-old Aire ${ }^{-/-}$mice and found a corroborating early age expansion in $\mathrm{CD} 11 \mathrm{~b}^{+} \mathrm{CD} 11 \mathrm{c}^{+} \mathrm{B}$ cells, which are thought to correspond phenotypically and functionally to human $\mathrm{CD} 21^{\mathrm{lo}} \mathrm{CD} 38^{\text {lo }} \mathrm{B}$ cells (Figure 4, E and F, and Supplemental Figure 8) (43-45). Similar expansion in $\mathrm{CD} 11 \mathrm{~b}^{+} \mathrm{CD} 11 \mathrm{c}^{+} \mathrm{B}$ cells was seen in the peripheral blood of 10 -week-old Aire ${ }^{-/-}$mice (data not shown). Collectively, APECED is associated with an altered peripheral blood immunophenotype with early expansion in $\mathrm{CD} 21^{10} \mathrm{CD} 38^{\text {lo }} \mathrm{B}$ lymphocytes.

\section{Discussion}

In this large prospectively evaluated cohort of American APECED patients, we found previously unappreciated early manifestations of the syndrome, with striking enrichment of organ-specific nonendocrine autoimmune manifestations. This study suggests that the spectrum of clinical features of APECED is broader and deeper and manifests earlier than previously appreciated and provides insight into how to accelerate diagnosis and allow earlier intervention.

By systematically defining early nonclassic triad APECED manifestations, we found that patients were likely to have been seen by dermatologists, gastroenterologists, dentists, ophthalmologists, pulmonologists, and hepatologists early in the course of their disease, often before they developed the widely recognized endocrine and fungal manifestations. In this patient cohort, incorporating urticarial eruption, intestinal dysfunction, and enamel hypoplasia into the diagnostic criteria of APECED would result in earlier recognition and diagnosis. Further research will be needed to validate the proposed expanded criteria in independent cohorts of APECED patients and to determine how many patients that are screened and meet the expanded diagnostic criteria do not end up developing APECED.

Although the proposed expanded criteria need to be validated further, it is of major clinical importance to follow up on this finding, since earlier recognition and diagnosis could have major implications for the prevention of episodes of life-threatening adrenal crisis and hypocalcemic seizures, which now represent the chief presentations of undiagnosed patients. Indeed, we show that, in this data set, approximately $40 \%$ of all patients would have been diagnosed by the proposed expanded diagnostic criteria but not the current classic diagnostic dyad before being hospitalized with hypocalcemic seizures and/or adrenal crises. Moreover, early diagnosis would provide a window for timely recognition and development of treatments for APECED's life-threatening autoimmune complications, including hepatitis and pneumonitis. Early diagnosis would allow consideration for preemptive immunomodulation before multiorgan autoimmunity develops, which might alter the natural history of APECED and avert the emergence of severe, often-irreversible, autoimmune complications, including those that have long-term adverse effects on bone metabolism, renal function, and fertility. Earlier diagnosis would also enable affected families to receive accurate genetic counseling and make informed reproductive decisions, when relevant.

Not all patients we identified as having APECED had biallelic AIRE mutations. Sanger sequencing did not universally validate the diagnosis in patients with suspected APECED. Between 15\% and 20\% of patients carry large AIRE deletions, heterozygous AIRE mutations, or even fully wild-type AIRE and yet 
present in ways clinically indistinguishable from those with proven biallelic AIRE mutations. This implies that non-AIRE genetic variants may cause APECED in some patients, presumably through the modulation of AIRE expression and/or through breakdown of AIRE-independent tolerance mechanisms.

Testing for anti-IFN- $\omega$ autoantibodies upon clinical suspicion may be helpful for diagnosis, as $97 \%$ of our patients had such autoantibodies, which are known to be highly prevalent and specific for APECED (13). In addition, testing for intrinsic factor antibodies and for autoantibodies against IL-17F/IL-22, IA-2, TPH, and BPIFB1/KCNRG may be helpful for predicting the development of B12 deficiency, CMC, type 1 diabetes, intestinal dysfunction, and pneumonitis, respectively. Moreover, the patient peripheral blood immunophenotypes showed increased $\mathrm{T}$ cells, CD4 lymphocytes, CD4/CD8 ratios, plasmablasts, and $\mathrm{CD} 21^{10} \mathrm{CD} 38^{\text {lo }} \mathrm{B}$ cells, which may be useful to confirm suspicion for APECED in patients with compatible clinical features. Future studies in larger numbers of patients will be required to examine the potential correlation between specific AIRE mutations and (a) the distribution of organ-specific or anticytokine autoantibodies and (b) the magnitude of specific immunological perturbations in affected patients. More research in AIRE-deficient humans and mice will also be required to define how lack of AIRE promotes these immunological alterations and how $\mathrm{T}$ and/or B cell-targeted immunomodulatory treatments affect disease outcomes.

In contrast to the European cohorts, which are more genetically and ethnically restricted, we found a striking enrichment of nonendocrine disorders in our patients. American patients suffered from a prominent hexad of urticarial eruption, hepatitis, gastritis, intestinal dysfunction, pneumonitis, and Sjögren's-like syndrome (40\%-80\%), uncommon entities in other cohorts (prevalences between $<5 \%$ and $\sim 20 \%$ ) (6-22, 46, 47). Of interest, carrying biallelic AIRE mutations other than the c.967_979del13 allele in heterozygosity or homozygosity was significantly associated with the time to development of urticarial eruption and hepatitis, indicating that the enrichment in some of the nonendocrine manifestations may be explained, at least in part, by the AIRE genotype. These observations broaden the spectrum of common nonendocrine manifestations affecting APECED patients and provide an opportunity (a) to examine the mechanisms by which AIRE protects from autoimmunity in the skin, liver, stomach, intestine, lungs, and salivary glands and (b) to further investigate potential genotype-phenotype correlations in APECED patients with a diverse genotypic signature.

Consistent prospective evaluation by a multidisciplinary team of specialists independent of disease manifestations allowed us to identify distinct disease features as well as those in common with other APECED cohorts. It is plausible that the uniform evaluation of all our patients by the same multidisciplinary team of specialists may have allowed us to unmask entities that are also common in European APECED cohorts but are challenging to systematically evaluate by single-specialty physicians. Alternatively, and/or in parallel, American APECED may indeed be a more phenotypically diverse syndrome; if so, genetic differences in the genetically diverse American population, along with environmental and/ or microbial factors, may account for the different phenotypic expression of organ-specific nonendocrine autoimmunity seen in the American cohort. Future enrollment and uniform multidisciplinary evaluation of European and additional American patients in our and other institutions will be essential to validate the proposed expanded diagnostic criteria and to define the full scope and underlying etiology of cohort-specific variations in phenotypic expression, beyond the previously described unique features of APECED in Iranian Jews (14).

In summary, our prospective multidisciplinary evaluation of patients with this complex multisystem disease afforded insights into its clinical presentation, diagnosis and pathogenesis. These findings redefine the clinical features and diagnostic criteria of APECED, thereby promoting its broader recognition and earlier diagnosis. More research is required to identify the yet-undefined genetic forms of APECED; to gain more insight into the organ-specific flaws in AIRE-dependent immune tolerance; to develop novel screening, diagnostic, and prognostic tools; and to implement targeted preventive and therapeutic strategies.

\section{Methods}

\section{Study participants}

Thirty-five consecutive APECED patients were enrolled (2013-2015) on a NIAID IRB-approved protocol and provided written informed consent. Inclusion criteria for enrollment consisted of either a clinical diagnosis of APECED based on development of any 2 manifestations within the classic triad of 
CMC, hypoparathyroidism, and adrenal insufficiency $(n=34)$ or a genetic diagnosis of APECED via identification of biallelic AIRE mutations but no development of a classic diagnostic dyad $(n=1)$ yet. Irrespective of disease manifestations, all patients underwent uniform prospective evaluation consisting of (a) blood, urine, and stool laboratory testing; (b) imaging studies (liver/renal ultrasounds, chest computed tomography); and (c) consultations by the same multidisciplinary team of specialists in infectious disease, immunology, endocrinology, hepatology, gastroenterology, pulmonology, nephrology, ophthalmology, dermatology, pediatrics, allergy, hematology, and dentistry, including Sjögren's evaluation. Clinical definitions, laboratory examinations, and statistical methods are detailed below. Whole blood was collected for flow cytometric lymphocyte analyses, and DNA was extracted for AIRE sequencing. Serum was obtained for autoantibody detection as described below. Healthy donors were enrolled in protocols approved by the NIAID, NIH Clinical Center, and NCI Institutional Review Board committees and provided written informed consent for study participation. The study was conducted in accordance with the Helsinki Declaration.

\section{AIRE sequencing}

DNA was harvested from whole blood using the Gentra Puregene Blood DNA isolation Kit (Qiagen) per the manufacturer's instructions. AIRE-coding exons were amplified by PCR using exon-specific oligonucleotide primers and GoTaq Hot Start Polymerase (Promega). The primers were identical for amplification and sequencing, and their sequences are provided in Supplemental Table 11. The PCR cycling conditions were as follows: $94^{\circ} \mathrm{C}$ for 2 minutes, followed by 30 cycles of $94^{\circ} \mathrm{C}$ for 30 seconds, $60^{\circ} \mathrm{C}$ for 30 seconds, and $72^{\circ} \mathrm{C}$ for 1 minute, followed by $4^{\circ} \mathrm{C}$ hold. Betaine (Sigma-Aldrich) was added in the PCR reactions at a final concentration of $1 \mathrm{M}$. Purified PCR products were directly sequenced with BigDye Terminators (version 1.1) and were analyzed on a 3130xL Genetic Analyzer (Applied Biosystems). Sanger sequencing cycling conditions were as follows: $96^{\circ} \mathrm{C}$ for 2 minutes, followed by 30 cycles of $96^{\circ} \mathrm{C}$ for 10 seconds and $60^{\circ} \mathrm{C}$ for 4 minutes, followed by $4^{\circ} \mathrm{C}$ hold.

In patients in whom initial Sanger sequencing did not reveal biallelic AIRE mutations, new amplification and sequencing primers were designed that cover AIRE exons, highly conserved intronic regions, and 5'UTR and 3'UTR regions (Supplemental Table 12). Specifically, 10-100 ng genomic DNA was amplified in a 15 - $\mu 1$ reaction consisting of $12.75 \mu 1$ Platinum Taq HiFi Supermix (Life Technologies), $0.75 \mu 1$ DMSO, and $0.4 \mu \mathrm{l}$ of each primer $\left(25 \mu \mathrm{M}\right.$ stock concentration). The PCR cycling conditions were as follows: $94^{\circ} \mathrm{C}$ for 3 minutes, followed by 35 cycles of $94^{\circ} \mathrm{C}$ for 20 seconds and $68^{\circ} \mathrm{C}$ for 2 minutes and 10 seconds, followed by $10^{\circ} \mathrm{C}$ hold. Sequencing reactions were performed in a $20-\mu 1$ reaction that contained $5 \%$ DMSO using BigDye Terminators v3.1 per the manufacturer's instructions at 1:8 strength. Reactions were purified on a DTR spin plate (Edge Biosystems), and reactions were run on an ABI3730XL. Chromatograms were aligned to reference sequence and analyzed using Sequencher v5.1.

Copy number variation was assessed in patients in whom biallelic AIRE mutations were not identified using both aforementioned sequencing methods by employing the exon-level oligo array CGH platform of GeneDx (ExonArrayDx; catalog 906). Data analysis was performed using gene-specific filtering. Probe sequences and locations were based on human genome build GRCh37/UCSC hg19. Confirmation of copy number changes was performed by repeat array CGH analysis. Array CGH alterations were reported according to the International System for Human Cytogenetic Nomenclature guidelines.

\section{Clinical definitions}

The diagnoses of hypoparathyroidism, adrenal insufficiency, primary hypothyroidism, primary hypogonadism, and type 1 diabetes mellitus were based on typical biochemical findings. In brief, the diagnosis of hypoparathyroidism was based on decreased plasma calcium, elevated plasma phosphate, and decreased or absent plasma intact parathyroid hormone. The diagnosis of adrenal insufficiency was based on decreased plasma cortisol concentrations upon adrenocorticotropic hormone (ACTH) stimulation. The diagnosis of primary hypothyroidism was based on decreased plasma-free thyroxine and elevated plasma thyroid-stimulating hormone. The diagnosis of primary ovarian failure was based on elevated plasma levels of follicle-stimulating hormone (FSH) or luteinizing hormone ( $\mathrm{LH})$, amenorrhea, and absent, slow, or regressing pubertal development. The diagnosis of testicular failure was based on decreased plasma levels of free testosterone and elevated plasma levels of FSH or LH. The diagnosis of type 1 diabetes mellitus was based on the presence of glycosuria, elevated plasma glucose levels, decreased plasma levels of insulin and 
C-peptide, and elevated hemoglobin A1c. GH deficiency was diagnosed based on decreased plasma levels of insulin-like growth factor-1 (IGF-1) and failure to respond to GH stimulation test.

Hepatitis was diagnosed based on chronic elevation of plasma transaminases with accompanying biopsy-proven evidence of lymphoplasmacytic hepatic inflammation without evidence of viral or drug-induced hepatitis. Gastritis was diagnosed based on endoscopic evaluation and biopsy-proven evidence of lymphocytic gastric inflammation. B12 deficiency was diagnosed based on decreased plasma levels of vitamin B12. Intestinal dysfunction was diagnosed based on the presence of either chronic nonbloody diarrhea or chronic constipation that required chronic administration of laxatives and/or enemas, or an alternating pattern of both, without meeting ROME III diagnostic criteria for irritable bowel syndrome. Accompanying symptoms included abdominal cramping/pain, and/or abdominal bloating/distention, and/or foul-smelling flatulence, and/or presence of floating/"greasy" stools. Tubulointerstitial nephritis was diagnosed based on biopsy-proven evidence of lymphocytic inflammation in the renal tubules and interstitium, accompanied by elevation in plasma creatinine levels. Pneumonitis was diagnosed based on the presence of chronic persistent cough and radiographic evidence of interstitial, nodular and/or ground-glass opacities, and/or bronchiectasis. The diagnosis was confirmed by biopsy-proven evidence of lymphocytic lung inflammation in 5 of 14 patients.

Sjögren's-like syndrome was diagnosed based on the presence of chronic sicca symptoms of dry mouth and/or dry eyes and/or objective assessment of dry mouth/eyes per the classification criteria proposed by the American-European Consensus Group (48). We named the entity Sjögren's-like syndrome instead of Sjögren's syndrome, because, although the minor salivary gland biopsies we were able to perform $(n=$ 4) histologically closely resembled the alterations observed in Sjögren's syndrome minor salivary gland biopsies (including a focus score $>1$ ), ENA autoantibodies were negative in APECED patients. Enamel hypoplasia was diagnosed by dental, clinical, and radiographic examination and was defined as a developmental disturbance of teeth, characterized by deficient or defective enamel matrix formation not attributed to dental caries. Keratoconjunctivitis was diagnosed based on the presence of photophobia and/or blurry vision, occurring with or without ocular dryness, along with evidence of decreased visual acuity and/or punctate corneal lesions on ophthalmologic examination.

CMC was diagnosed based on recurrent episodes of Candida infections of the mucous membranes, skin, and/or nails. The diagnosis of oral candidiasis was based on visible oral mucosal plaques and positive Candida cultures. The diagnosis of esophageal candidiasis was based on endoscopic evaluation and biopsy-proven evidence of fungal esophagitis with positive Candida cultures. The diagnosis of vulvovaginal candidiasis was based on the presence of vaginal symptoms, with accompanying demonstration of Candida on vaginal smear and/or positive Candida cultures, per the IDSA/CDC guidelines $(49,50)$. The diagnosis of skin and nail candidiasis was based on corresponding scrapings and positive Candida cultures. The diagnosis of candidiasis was further confirmed by the clinical response obtained after initiation of targeted antifungal therapy.

Nail dystrophy without candidiasis was characterized by pitted nail changes without positive Candida cultures and a lack of clinical response to targeted antifungal therapy. Alopecia was diagnosed based on thinning or complete loss of hair, either in the scalp and extremities (alopecia areata) or throughout the body (alopecia totalis). Asplenia was diagnosed based on the visualization of atrophic or absent spleen in abdominal ultrasound and/or computed tomography and of Howell-Jolly bodies in peripheral blood smears. Early-onset hypertension was defined as onset of elevated blood pressure before the age of 40 years and was accompanied by hypokalemia without evidence of mineralocorticoid excess, as previously described (6).

\section{Autoantibody detection}

Luciferase immunoprecipitation systems assay. The luciferase immunoprecipitation systems (LIPS) immunoassay technology was used to detect autoantibody immunoreactivity in the APECED cohort (51). Several of the LIPS autoantigens employed in this study have previously been described, including IA-2, tyrosine hydroxylase, IFN- $\omega$, and KCNRG (51-56). In addition, 4 new autoantigens, TPH, SCC, NLR family and pyrin domain containing 5 (NALP5), and BPIFB1, were generated as mammalian expression vectors for expression as fusion protein with luciferase. DNA fragments encoding TPH, SCC, and NALP5 were subcloned downstream of Renilla luciferase into the BamH1-XhoI site using the pREN2 vector (57). For BPIFB1, the Gaussia luciferase vector (pGAUS3) was utilized for expression as an N-terminal fusion 
protein containing its signal sequence. DNA sequencing was used to confirm the integrity of the 3 new constructs.

LIPS testing was performed as previously described (58). In brief, serum samples from the APECED patients $(n=35)$ and healthy donor subjects $(n=10)$ were first diluted $1: 10$ in assay buffer A $(20 \mathrm{mM}$ Tris, $\mathrm{pH} 7.5,150 \mathrm{mM} \mathrm{NaCl}, 5 \mathrm{mM} \mathrm{MgCl}_{2}, 1 \%$ Triton X-100) to generate a master plate. As described in a detailed publication and corresponding video (58), LIPS testing was initiated by adding $40 \mu 1$ of buffer A, $10-\mu 1$ aliquots of serum (equivalent to $1 \mu 1$ of serum) from the master plate, and $50 \mu 1$ of each luciferase antigen. Cos 1 cell extract typically containing an equivalent of $10^{7}$ light units (LU) was added to a polypropylene plate. After 1-hour incubation at room temperature with shaking, the mixture containing IgG antibody-antigen complexes was transferred to a microtiter filter plate containing protein $A / G$ beads for incubation for 1 additional hour. Next, the filter plate was washed with buffer on a vacuum manifold to remove unbound luciferase-tagged antigens. LU were then measured using a luminometer following the addition of coelenterazine substrate. All LU data were obtained from the average of at least 2 separate experiments. The prevalence of these autoantibodies in the APECED cohort was calculated based on the means plus 3 standard deviations of the control group.

Particle-based multiplex assay. Serum or plasma from the APECED patients $(n=35)$ and healthy control subjects $(n=100)$ enrolled through the NIH Blood Bank were screened for anticytokine autoantibodies using a particle-based approach as previously described (59). In brief, differentially fluorescing magnetic beads (Bio-Rad) were covalently coupled to $2.5 \mu \mathrm{g}$ of recombinant human IFN- $\alpha$, IFN- $\gamma$, granulocyte macrophage colony-stimulating factor (GM-CSF), IL- $\alpha$, IL-12p70, IL-17A, IL-17F, or IL-22 (eBioscience). Beads were combined and incubated with patient or control serum or plasma (1:100 dilution) for 30 minutes, washed, and then incubated with PE-labeled goat anti-human IgG (1 $\mu \mathrm{g} / \mathrm{ml}$, eBioscience) for an additional 30 minutes. Beads were washed again and run in a multiplex fashion on the Bio-Plex 200 (Bio$\mathrm{Rad}$ ) instrument, and fluorescence intensity for each bead was plotted as a function of autoantibody titer (GraphPad Prism version 6). The prevalence of these autoantibodies in the APECED cohort was calculated based on the means plus 3 standard deviations of the control group.

Other methodologies. Chemiluminescence was employed to measure autoantibodies targeted against thyroid peroxidase and anti-thyroglobulin, radioluminescence was used to measure autoantibodies targeted against GAD65, immunoabsorption was used to measure autoantibodies targeted against 21-hydroxylase, and enzyme immunoassay was used to measure autoantibodies targeted against ENA in the sera of the APECED patients (60-62). These tests were performed at the NIH Clinical Center Department of Laboratory Medicine.

Intrinsic factor blocking antibody and parietal cell IgG antibody determination Intrinsic factor blocking antibody and parietal cell IgG antibody measurements in the sera of APECED patients were performed at the NIH Clinical Center Department of Laboratory Medicine using a competitive binding immunoenzymatic assay and enzyme-linked immunosorbent assay, respectively.

\section{Lymphocyte immunophenotyping of human blood using flow cytometry}

Whole blood was harvested into EDTA tubes, red blood cells were lysed using BD FACS Lysing Solution (BD Biosciences), and staining was performed using fluorochrome-conjugated (FITC, PE, PE-Cy7, APC, APC-eFluor 780, Alexa Fluor 700, eFluor 450, PerCP-Cy5.5) antibodies against human CD45 (HI30), CD19 (SJ25C1), CD27 (TNFRSF7), and CD10 (CB-CALLA) (eBioscience); CD3 (SK7), CD16 (SK7), CD56 (SK7), CD20 (L27), CD62L (SK11), and CD38 (HB7) (BD Biosciences); IgM (MHM-88) and CD21 (Bu32) (BioLegend); CD4 (S3.5) and CD8 (3B5) (ThermoFisher Scientific); and CD45RA (ALB11) (Beckman Coulter) for 30 minutes on ice. After incubation, the cells were washed with FACS buffer and the samples were acquired using a FACSCanto (BD Biosciences). FlowJo (TreeStar) was used for the final analysis. Cell numbers were quantified from the FACS plot percentages using the patient's same-day absolute lymphocyte count obtained from the complete blood cell with white count differential.

\section{Histology}

Liver, stomach, lung, and minor salivary gland biopsies from APECED patients with hepatitis, gastritis, pneumonitis, and Sjögren's-like syndrome, respectively, were routinely processed and sectioned at $5 \mu \mathrm{m}$ for H\&E. Skin biopsies from APECED children with urticarial eruption were routinely processed and sectioned at $5 \mu \mathrm{m}$ for $\mathrm{H} \& \mathrm{E}$ and immunohistochemistry. Immunohistochemistry was performed at the 
NCI Laboratory of Pathology using the Ventana Benchmark Ultra automated IHC/ISH slide staining system (Ventana Medical Systems, Inc.) and Ultra view detection system (Roche). The primary antibodies used were CD3 (prediluted, clone 2GV6; Ventana Medical Systems), CD4 (prediluted, clone SP35; Ventana Medical Systems), CD8 (1:25 dilution, clone CD8/144B; Dako), CD79a (prediluted, clone SP18; Ventana Medical Systems), CD68 (prediluted, clone KP-1; Ventana Medical Systems), CD163 (prediluted, clone MRQ-26; Ventana Medical Systems), and myeloperoxidase (MPO; 1:1,000 dilution, rabbit polyclonal; Dako).

\section{B cell immunophenotyping of mouse blood using flow cytometry}

Air $^{-/-}$mice in the NOD genetic background, developed as previously described (1), were purchased from The Jackson Laboratories. Five- to six- and ten-week-old sex-matched Aire ${ }^{+/+}$and Aire Al- $^{-1}$ litermates were used to determine the frequency and numbers of $\mathrm{CD} 11 \mathrm{c}^{+} \mathrm{CD} 11 \mathrm{~b}^{+} \mathrm{B}$ cells in mouse peripheral blood. The mice were anesthetized using ketamine/xylazine and blood was obtained via cardiac puncture in EDTA-containing tubes. To obtain single cell suspensions from blood, red blood cells were lysed with BD Pharmlyse lysis buffer (BD Biosciences), according to the manufacturer's instructions, and the leukocytes were washed with HBSS containing $2 \mathrm{mM}$ EDTA and 1\% BSA, resuspended in PBS, and filtered as previously described (63). Cell suspensions were initially stained with a fluorescent LIVE/DEAD dye (Invitrogen) followed by blocking with rat anti-mouse CD16/32 (eBioscience) and surface staining with fluorochrome-conjugated (FITC, PE, PerCPCy5.5, PerCP-eFluor710, APC, APCeFluor 780, or eFluor450) antibodies against mouse CD45 (clone 30-F11), MHCII (anti-rat RT1B; OX-6), CD11b (M1/70), CD11c (N418), CD3e (145-2C11), NKp46 (29A1.4), and CD19 (1D3). FACS was performed on a 5-laser LSRFortessa (BD Biosciences), and the data were analyzed using Flowjo software (Tree Star). Only single cells were analyzed, and cell numbers were quantified using PE-conjugated fluorescent counting beads (Spherotech), as previously described (63).

\section{Statistics}

Log-rank tests were employed to compare time to development of a clinical manifestation by autoantibody presence and specific AIRE mutant alleles, using the R statistical software package (64). Log-rank tests were used because patients were of different ages and, therefore, the clinical manifestation(s) could present at different times. Although we acknowledge that many comparisons were made, we only considered variables with a $P$ value of less than 0.05 to be of potential interest for further investigation.

Comparison of the frequency and/or absolute numbers of different lymphocyte subsets between APECED patients and healthy donors and between Aire $^{+/+}$mice and Air ${ }^{-/-}$mice were performed using a 2-tailed unpaired $t$ test (with Welch's correction, where necessary) or Mann-Whitney $U$ test, where appropriate, using GraphPad Prism 6.0 and were presented as mean \pm SEM. A $P$ value of less than 0.05 was considered statistically significant.

\section{Study approval}

APECED patients and healthy donors were enrolled in protocols approved by the NIAID, NIH Clinical Center, and NCI Institutional Review Board committees and provided written informed consent for study participation. Written informed consent was provided for patient photographs appearing in the manuscript. The study was conducted in accordance with the Helsinki Declaration.

Animal studies were performed in accordance with the recommendations in the Guide for the Care and Use of Laboratory Animals (National Academies Press. 2011) and under the auspices of protocol LCID14E, which was approved by the Animal Care and Use Committee of the NIAID.

\section{Author contributions}

EMNF, SRR, and MSL wrote the manuscript; EMNF, SRR, TH, KKW, and MSL designed the research studies; EMNF, SRR, HHK, DC, TS, AP, AB, MN, IA, NMM, AS, MNS, DML, KDS, JBK, RJB, SMH, KNO, TAF, TH, KKW, and MSL evaluated patients and provided patient care; EMNF, SRR, AB, MN, LY, CF, and MSL coordinated the conduct of clinical research studies; SDR, PDB, KRR, JEN, LBR, TJB, SKB, APH, MS, and ALC conducted experiments; EMNF, SRR, SDR, PDB, KRR, JEN, LBR, TJB, SKB, and APH acquired data; and EMNF, SRR, SDR, PDB, KRR, JEN, LBR, TJB, WG, SH, SKB, APH, SR, HHK, CCRL, AB, MN, JM, DEK, MQ, IA, KRC, JA, GU, TAF, and MSL analyzed data. 


\section{Acknowledgments}

We are grateful to our patients and their families for their participation in our study and to the NIAID clinical fellows, residents, and nursing staff for their dedication to patient care. This work is dedicated to the family of C.D., our 14-year-old APECED patient who succumbed to pneumonitis-related respiratory failure. This research was supported by the Division of Intramural Research of the NIH, USA; the NIAID; the NIH Clinical Center; the National Institute of Dental and Craniofacial Research; the NCI; the National Institute of Diabetes and Digestive and Kidney Diseases; the National Human Genome Research Institute; the National Eye Institute; the National Heart, Lung, and Blood Institute; and the Eunice Kennedy Shriver National Institute of Child Health and Human Development. This project was also funded in part with federal funds from the NCI, NIH, under contract no. HHSN261200800001E. The content of this publication does not necessarily reflect the views or policies of the Department of Health and Human Services, nor does mention of trade names, commercial products, or organizations imply endorsement by the US government.

Address correspondence to: Michail S. Lionakis, Fungal Pathogenesis Unit, Laboratory of Clinical Infectious Diseases (LCID), National Institute of Allergy and Infectious Diseases, National Institutes of Health, 9000 Rockville Pike, Building 10, Room 11C102, Bethesda, Maryland 20892, USA. Phone: 301.443.5089; E-mail: lionakism@mail.nih.gov.

SRR's present address is: Division of Infectious Diseases, Baylor College of Medicine, Houston, Texas, USA.

SKB's present address is: Division of Vaccines and Related Product Applications, Center for Biologics Evaluation and Research, US Food and Drug Administration, Silver Spring, Maryland, USA.

1. Anderson MS, et al. Projection of an immunological self shadow within the thymus by the aire protein. Science. 2002;298(5597):1395-1401.

2. Mathis D, Benoist C. Aire. Annu Rev Immunol. 2009;27:287-312.

3. Oftedal BE, et al. Dominant mutations in the autoimmune regulator AIRE are associated with common organ-specific autoimmune diseases. Immunity. 2015;42(6):1185-1196.

4. Cetani F, et al. A novel mutation of the autoimmune regulator gene in an Italian kindred with autoimmune polyendocrinopathy-candidiasis-ectodermal dystrophy, acting in a dominant fashion and strongly cosegregating with hypothyroid autoimmune thyroiditis. J Clin Endocrinol Metab. 2001;86(10):4747-4752.

5. Su MA, et al. Mechanisms of an autoimmunity syndrome in mice caused by a dominant mutation in Aire. J Clin Invest. 2008;118(5):1712-1726.

6. Ahonen P, Myllärniemi S, Sipilä I, Perheentupa J. Clinical variation of autoimmune polyendocrinopathy-candidiasis-ectodermal dystrophy (APECED) in a series of 68 patients. N Engl J Med. 1990;322(26):1829-1836.

7. Perheentupa J. Autoimmune polyendocrinopathy-candidiasis-ectodermal dystrophy. J Clin Endocrinol Metab. 2006;91(8):2843-2850.

8. Wolff AS, et al. Autoimmune polyendocrine syndrome type 1 in Norway: phenotypic variation, autoantibodies, and novel mutations in the autoimmune regulator gene. J Clin Endocrinol Metab. 2007;92(2):595-603.

9. Husebye ES, Perheentupa J, Rautemaa R, Kämpe O. Clinical manifestations and management of patients with autoimmune polyendocrine syndrome type I. J Intern Med. 2009;265(5):514-529.

10. Dominguez M, et al. Autoimmune polyendocrinopathy-candidiasis-ectodermal dystrophy (APECED) in the Irish population. J Pediatr Endocrinol Metab. 2006;19(11):1343-1352.

11. F Magitta N, et al. Autoimmune polyendocrine syndrome type I in Slovakia: relevance of screening patients with autoimmune Addison's disease. Eur J Endocrinol. 2008;158(5):705-709.

12. Meloni A, et al. Autoimmune polyendocrine syndrome type 1: an extensive longitudinal study in Sardinian patients. J Clin Endocrinol Metab. 2012;97(4):1114-1124.

13. Meager A, et al. Anti-interferon autoantibodies in autoimmune polyendocrinopathy syndrome type 1. PLoS Med. 2006;3(7):e289.

14. Zlotogora J, Shapiro MS. Polyglandular autoimmune syndrome type I among Iranian Jews. J Med Genet. 1992;29(11):824-826.

15. Orlova EM, et al. Autoimmune polyglandular syndrome type 1 in Russian patients: clinical variants and autoimmune regulator mutations. Horm Res Paediatr. 2010;73(6):449-457.

16. Pearce SH, et al. A common and recurrent 13-bp deletion in the autoimmune regulator gene in British kindreds with autoimmune polyendocrinopathy type 1. Am J Hum Genet. 1998;63(6):1675-1684.

17. Myhre AG, et al. Autoimmune polyendocrine syndrome type 1 (APS I) in Norway. Clin Endocrinol (Oxf). 2001;54(2):211-217.

18. Neufeld M, Maclaren NK, Blizzard RM. Two types of autoimmune Addison's disease associated with different polyglandular autoimmune (PGA) syndromes. Medicine (Baltimore). 1981;60(5):355-362.

19. Podkrajsek KT, Bratanic N, Krzisnik C, Battelino T. Autoimmune regulator-1 messenger ribonucleic acid analysis in a novel intronic mutation and two additional novel AIRE gene mutations in a cohort of autoimmune polyendocrinopathy-candidiasis-ectodermal dystrophy patients. J Clin Endocrinol Metab. 2005;90(8):4930-4935. 
20. Stolarski B, et al. Molecular background of polyendocrinopathy-candidiasis-ectodermal dystrophy syndrome in a Polish population: novel AIRE mutations and an estimate of disease prevalence. Clin Genet. 2006;70(4):348-354.

21. Valenzise M, et al. Autoimmune polyendocrinopathy-candidiasis-ectodermal dystrophy: report of seven additional sicilian patients and overview of the overall series from sicily. Horm Res Paediatr. 2014;82(2):127-132.

22. Bruserud $\varnothing$, et al. A longitudinal follow-up of Autoimmune polyendocrine syndrome type 1 [published online ahead of print June 2, 2016]. J Clin Endocrinol Metab. doi:10.1210/jc.2016-1821.

23. Popler J, et al. Autoimmune polyendocrine syndrome type 1: Utility of KCNRG autoantibodies as a marker of active pulmonary disease and successful treatment with rituximab. Pediatr Pulmonol. 2012;47(1):84-87.

24. Huibregtse KE, Wolfgram P, Winer KK, Connor EL. Polyglandular autoimmune syndrome type I - a novel AIRE mutation in a North American patient. J Pediatr Endocrinol Metab. 2014;27(11-12):1257-1260.

25. Friedman TC, et al. Frequent occurrence of asplenism and cholelithiasis in patients with autoimmune polyglandular disease type I. Am J Med. 1991;91(6):625-630.

26. Bøe Wolff AS, et al. AIRE variations in Addison's disease and autoimmune polyendocrine syndromes (APS): partial gene deletions contribute to APS I. Genes Immun. 2008;9(2):130-136.

27. Heino M, et al. Mutation analyses of North American APS-1 patients. Hum Mutat. 1999;13(1):69-74.

28. Winqvist O, Gustafsson J, Rorsman F, Karlsson FA, Kämpe O. Two different cytochrome P450 enzymes are the adrenal antigens in autoimmune polyendocrine syndrome type I and Addison's disease. J Clin Invest. 1993;92(5):2377-2385.

29. Winqvist O, Karlsson FA, Kämpe O. 21-Hydroxylase, a major autoantigen in idiopathic Addison's disease. Lancet. 1992;339(8809):1559-1562.

30. Puel A, et al. Autoantibodies against IL-17A, IL-17F, and IL-22 in patients with chronic mucocutaneous candidiasis and autoimmune polyendocrine syndrome type I. J Exp Med. 2010;207(2):291-297.

31. Kisand $\mathrm{K}$, et al. Chronic mucocutaneous candidiasis in APECED or thymoma patients correlates with autoimmunity to Th17-associated cytokines. J Exp Med. 2010;207(2):299-308.

32. Ekwall O, et al. Identification of tryptophan hydroxylase as an intestinal autoantigen. Lancet. 1998;352(9124):279-283.

33. Shum AK, et al. BPIFB1 is a lung-specific autoantigen associated with interstitial lung disease. Sci Transl Med. 2013;5(206):206ra139.

34. Alimohammadi M, et al. Pulmonary autoimmunity as a feature of autoimmune polyendocrine syndrome type 1 and identification of KCNRG as a bronchial autoantigen. Proc Natl Acad Sci U S A. 2009;106(11):4396-4401.

35. Perniola R, Lobreglio G, Rosatelli MC, Pitotti E, Accogli E, De Rinaldis C. Immunophenotypic characterisation of peripheral blood lymphocytes in autoimmune polyglandular syndrome type 1: clinical study and review of the literature. J Pediatr Endocrinol Metab. 2005;18(2):155-164.

36. Sedivá A, Ciháková D, Lebl J. Immunological findings in patients with autoimmune polyendocrinopathy-candidiasis-ectodermal dystrophy (APECED) and their family members: are heterozygotes subclinically affected?. J Pediatr Endocrinol Metab. 2002;15(9):1491-1496.

37. Wolff AS, Oftedal BE, Kisand K, Ersvaer E, Lima K, Husebye ES. Flow cytometry study of blood cell subtypes reflects autoimmune and inflammatory processes in autoimmune polyendocrine syndrome type I. Scand J Immunol. 2010;71(6):459-467.

38. Isnardi I, et al. Complement receptor 2/CD21- human naive B cells contain mostly autoreactive unresponsive clones. Blood. 2010;115(24):5026-5036.

39. Wehr C, et al. The EUROclass trial: defining subgroups in common variable immunodeficiency. Blood. 2008;111(1):77-85.

40. Warnatz K, et al. Expansion of CD19(hi)CD21(lo/neg) B cells in common variable immunodeficiency (CVID) patients with autoimmune cytopenia. Immunobiology. 2002;206(5):502-513.

41. Kuehn HS, et al. Immune dysregulation in human subjects with heterozygous germline mutations in CTLA4. Science. 2014;345(6204):1623-1627.

42. Wehr C, et al. A new CD21low B cell population in the peripheral blood of patients with SLE. Clin Immunol. 2004;113(2):161-171

43. Rubtsov AV, Rubtsova K, Kappler JW, Jacobelli J, Friedman RS, Marrack P. CD11c-Expressing B Cells Are Located at the T Cell/B Cell Border in Spleen and Are Potent APCs. J Immunol. 2015;195(1):71-79.

44. Rubtsov AV, et al. Toll-like receptor 7 (TLR7)-driven accumulation of a novel CD11c+ B-cell population is important for the development of autoimmunity. Blood. 2011;118(5):1305-1315.

45. Hao Y, O'Neill P, Naradikian MS, Scholz JL, Cancro MP. A B-cell subset uniquely responsive to innate stimuli accumulates in aged mice. Blood. 2011;118(5):1294-1304.

46. Kisand K, Peterson P. Autoimmune polyendocrinopathy candidiasis ectodermal dystrophy. J Clin Immunol. 2015;35(5):463-478.

47. Jääskeläinen J, Perheentupa J. Autoimmune polyendocrinopathy-candidosis-ectodermal dystrophy (APECED)--a diagnostic and therapeutic challenge. Pediatr Endocrinol Rev. 2009;7(2):15-28.

48. Vitali C, et al. Classification criteria for Sjögren's syndrome: a revised version of the European criteria proposed by the American-European Consensus Group. Ann Rheum Dis. 2002;61(6):554-558.

49. Pappas PG, et al. Clinical practice guidelines for the management of candidiasis: 2009 update by the Infectious Diseases Society of America. Clin Infect Dis. 2009;48(5):503-535.

50. Vulvovaginal Candidiasis. Center for Disease Control and Prevention. http://www.cdc.gov/std/tg2015/candidiasis.htm. Updated June 4, 2015. Accessed July 19, 2016.

51. Burbelo PD, Ching KH, Bush ER, Han BL, Iadarola MJ. Antibody-profiling technologies for studying humoral responses to infectious agents. Expert Rev Vaccines. 2010;9(6):567-578.

52. Burbelo PD, et al. A new luminescence assay for autoantibodies to mammalian cell-prepared insulinoma-associated protein 2. Diabetes Care. 2008;31(9):1824-1826.

53. Burbelo PD, et al. Extrapancreatic autoantibody profiles in type I diabetes. PLoS One. 2012;7(9):e45216.

54. Burbelo PD, et al. Anti-cytokine autoantibodies are associated with opportunistic infection in patients with thymic neoplasia. Blood. 2010;116(23):4848-4858.

55. Ching KH, et al. Two major autoantibody clusters in systemic lupus erythematosus. PLoS One. 2012;7(2):e32001.

56. Burbelo PD, et al. Rapid induction of autoantibodies during ARDS and septic shock. J Transl Med. 2010;8:97. 
57. Burbelo PD, Goldman R, Mattson TL. A simplified immunoprecipitation method for quantitatively measuring antibody responses in clinical sera samples by using mammalian-produced Renilla luciferase-antigen fusion proteins. BMC Biotechnol. 2005;5:22.

58. Burbelo PD, Ching KH, Klimavicz CM, Iadarola MJ. Antibody profiling by Luciferase Immunoprecipitation Systems (LIPS). JVis Exp. 2009;(32):1549.

59. Ding L, Mo A, Jutivorakool K, Pancholi M, Holland SM, Browne SK. Determination of human anticytokine autoantibody profiles using a particle-based approach. J Clin Immunol. 2012;32(2):238-245.

60. Kaczur V, et al. Effect of anti-thyroid peroxidase (TPO) antibodies on TPO activity measured by chemiluminescence assay. Clin Chem. 1997;43(8 Pt 1):1392-1396.

61. Walikonis JE, Lennon VA. Radioimmunoassay for glutamic acid decarboxylase (GAD65) autoantibodies as a diagnostic aid for stiff-man syndrome and a correlate of susceptibility to type 1 diabetes mellitus. Mayo Clin Proc. 1998;73(12):1161-1166.

62. Tanaka H, et al. Steroid 21-hydroxylase autoantibodies: measurements with a new immunoprecipitation assay. J Clin Endocrinol Metab. 1997;82(5):1440-1446.

63. Lionakis MS, Lim JK, Lee CC, Murphy PM. Organ-specific innate immune responses in a mouse model of invasive candidiasis. J Innate Immun. 2011;3(2):180-199.

64. R Core Team (2014). The R Project for Statistical Computing. http://www.R-project.org. Accessed July $19,2016$. 